\title{
Orientação nutricional de crianças e adolescentes e os novos padrões de consumo: propagandas, embalagens e rótulos
}

\author{
Nutritional guidance for children and adolescents and the new consumption patterns: \\ advertising, packaging and labeling
}

Tatiana Elias Pontes ${ }^{1}$, Thalita Feitosa Costa' ${ }^{1}$ Annete Bressan R. F. Marum², Anne Lise D. Brasil' ${ }^{3}$ José Augusto de A. C. Taddei

\section{RESUMO}

Objetivo: Compilar e debater conhecimentos sobre os rótulos, as embalagens e a divulgação e promoção de alimentos processados que progressivamente passam a compor a dieta da população infanto-juvenil.

Fontes de dados: Artigos nos bancos de dados eletrônicos, Medline e SciELO nos últimos dez anos, nas línguas portuguesa e inglesa, utilizando os descritores "criança”, "marketing", "hábitos alimentares", "televisão", "educação em Saúde". Também foram consultados livros, textos recentes e artigos considerados relevantes para realização dessa revisão.

Síntese dos dados: $\mathrm{O}$ artigo discute o marketing de alimentos, segundo os tópicos: propagandas, embalagens e rótulos. Quanto à publicidade televisiva, foi abordada sua influência na formação dos hábitos alimentares infantojuvenis, a qualidade nutricional dos alimentos veiculados e as alternativas para que pais e educadores possam lidar com os novos padrões de consumo. Além disso, debateram-se as embalagens enquanto meios de comunicação entre fabricante e consumidor. Sobre os rótulos, foram abordadas as novas regras da Agência Nacional de Vigilância Sanitária a respeito da Rotulagem Nutricional Obrigatória, bem como a importância da leitura adequada para a adoção de escolhas alimentares saudáveis.

Conclusões: Espera-se que este artigo seja instrumento de atualização dos profissionais da Saúde, proporcionando atuação mais incisiva no processo de educação em saúde e nutrição. Torna-se urgente a adoção de práticas preventivas com esclarecimento aos pais e familiares cujos filhos participam ativamente da sociedade de consumo, visando minimizar os efeitos deletérios da ingestão rotineira de alimentos obesogênicos.

Palavras-chave: criança; propaganda; hábitos alimentares; televisão; educação em saúde.

\section{ABSTRACT}

Objective: To debate knowledge on labeling, packaging, releases and promotion of processed foods that have been included progressively in the diets of children and adolescents.

Data source: On-line articles from Medline and SciELO database published over the last ten years in Portuguese and English using the keywords "child", "marketing”, "eating habits", "television", "health education". Also, books, recent texts and articles considered relevant for the present review were included.

Data synthesis: The present article discusses the food marketing according to propaganda, packaging and labeling. This review also approaches the influence of TV advertising on building of children and adolescents' eating habits; the nutrition quality of advertised foods and alternatives that parents and teachers could use to deal with these new patterns of consumption. Additionally, it is discussed packaging as a communication tool between manufacturer
Instituição: Departamento de Pediatria da Universidade Federal de São Paulo - Escola Paulista de Medicina (Unifesp-EPM), São Paulo, SP, Brasil ${ }^{1}$ Graduandas em Medicina pela Unifesp-EPM, São Paulo, SP, Brasil ${ }^{2}$ Mestre em Nutrição pela Unifesp-EPM, São Paulo, SP, Brasil

${ }^{3}$ Doutora em Pediatria, médica assistente da Disciplina de Nutrologia do Departamento de Pediatria da Unifesp-EPM, São Paulo, SP, Brasil

${ }^{4}$ Professor-associado da Disciplina de Nutrologia do Departamento de Pediatria da Unifesp-EPM, São Paulo, SP, Brasil
Endereço para correspondência:

José Augusto de A. C. Taddei

Rua Loefgreen, 1.647

CEP 04040-032 - São Paulo/SP

E-mail: taddei.dped@epm.br/nutsec@yahoo.com.br

Fonte financiadora do projeto: Conselho Nacional de Desenvolvimento Científico e Tecnológico (CNPq)

Recebido em: 6/5/08

Aprovado em: 1/9/08 
and consumer. Regarding labeling, the "Agência Nacional de Vigilância Sanitária” (Anvisa) new rules on Regulatory Nutritional Labeling as well as the importance of proper label reading for healthy choices are discussed.

Conclusions: The present article is intended to be a tool for updating health professionals and encouraging them to act more effectively in health education and nutrition. It is urgent to adopt prevention practices enlightening parents and relatives whose children actively participate in the consumption society in order to minimize the harmful effects of the obesogenic foods routine ingestion.

Key-words: child; propaganda; food habits; television; health education.

\section{Introdução}

O profissional de saúde que faz a orientação nutricional de crianças e adolescentes precisa se informar sobre rotulagem, divulgação e promoção de alimentos processados que, progressivamente, passam a compor a dieta da população infanto-juvenil.

O crescimento geométrico de alternativas alimentares que caracterizam a sociedade pós-moderna traz em si grandes vantagens nutricionais ao facilitar o transporte, armazenamento e preparo de refeições para crianças e adolescentes. São grandes as possibilidades de enriquecimento com micronutrientes, pró e pré-bióticos, assim como as oportunidades de formulação de alimentos que levem a mais conforto, prazer e melhores níveis de saúde.

Tal crescimento, no entanto, traz em seu bojo algumas influências negativas que vêm piorando o padrão de consumo de crianças e adolescentes. A alimentação inadequada está vinculada ao estímulo de alimentos em quantidade excessiva e qualidade inadequada, com excesso de açúcares, sódio, gorduras e deficiência de fibras e micronutrientes.

A possibilidade de orientação da população quanto ao consumo adequado de alimentos pode corrigir erros alimentares, diminuir seus efeitos deletérios e, simultaneamente, promover o redirecionamento da oferta de alimentos pelo setor produtivo à sociedade de consumo e seus mecanismos de divulgação.

Nesse contexto, deseja-se fornecer instrumentos ao profissional de saúde que atende à criança e ao adolescente, aprimorando a abordagem dos temas em questão na prática clínica. As informações presentes nessa revisão resultam da compilação e análise crítica de artigos obtidos nos bancos de dados eletrônicos Medline e SciELO nos últimos dez anos, nas línguas portuguesa e inglesa, bem como de livros e textos recentes.

Espera-se que, com as considerações subsequentes, estes profissionais tornem-se aptos a participar ativamente do processo de educação em saúde e nutrição, orientando adequadamente seus paciente e familiares sobre como proceder a uma leitura mais crítica das propagandas, embalagens e rótulos de alimentos, com ciência do forte impacto do consumo consciente na qualidade de vida a longo prazo.

\section{A participação do marketing no novo padrão de consumo alimentar}

A formação dos hábitos alimentares se processa gradualmente, principalmente durante a primeira infância, de forma que quaisquer inadequações devem ser retificadas no tempo apropriado sob orientação correta. Nesse processo, também estão envolvidos valores culturais, sociais, afetivos ou emocionais e comportamentais, de modo que se tornou crescente a percepção de que existe grande diferença entre comer (um ato social) e nutrir-se (uma atividade biológica) ${ }^{(1)}$.

A criança exerce um papel ativo desde os primeiros anos de vida, quando já é capaz de influenciar os cuidados e as relações familiares de que participa. É um processo que ocorre dentro das relações bidirecionais, em que a criança influencia e é influenciada por aqueles ao seu redor ${ }^{(2)}$.

A mídia televisiva tem participação ativa e majoritária nas atividades prosaicas infanto-juvenis; assim, os meios de comunicação acabam por desempenhar papel estruturador na construção e desconstrução de hábitos e práticas alimentares ${ }^{(3)}$.

Logo, a propaganda agrada às emoções, não ao intelecto, afetando mais profundamente crianças que adultos; o falso conceito de alimento como algo que dê poder é perigoso por permitir que as indústrias alimentícias explorem a vulnerabilidade das crianças ${ }^{(4)}$.

\section{O marketing alimentício na TV}

Almeida, Nascimento e Quaioti ${ }^{(5)}$ realizaram uma análise qualitativa do marketing alimentício e reconstruíram a pirâmide alimentar a partir das propagandas analisadas. Diferentemente da pirâmide considerada ideal, 60\% concentraram-se no grupo de gorduras, óleos e doces, com consequente redução do grupo de pães, cereais e massas, 
além da ausência de carboidratos complexos (frutas e vegetais in natura) ${ }^{(5)}$.

Em geral, tal padrão nutricional é mantido, de modo que a maioria dos alimentos veiculados em propagandas televisivas é de alta densidade energética, ricos em sódio, gorduras e açúcares, e pobres em fibras alimentares e carboidratos complexos ${ }^{(6,7)}$.

\section{Brincadeiras e atividades físicas: promoção de saúde}

Quaisquer intervenções feitas por profissionais da saúde devem levar em conta a adoção de hábitos alimentares saudáveis, estando inseridas em um amplo contexto de promoção de saúde e adaptadas à realidade social infanto-juvenil, atuando sobretudo no ambiente familiar e escolar.

No ambiente familiar é fundamental que os pais participem ativamente das escolhas alimentares de seus filhos desde a primeira infância. Participar deste processo, no entanto, é muito mais do que o preparo da comida; é preconizar que as refeições sejam feitas à mesa, tornando aquele momento prazeroso à criança e ao adolescente.

Por outro lado, vale salientar a importância dos pais na adoção, ou não, de hábitos saudáveis. Durante o desenvolvimento neuropsicomotor da criança, a figura materna e paterna é tida como referenciais a serem seguidos e imitados. Assim, escolhas nutricionalmente adequadas serão incorporadas aos comportamentos alimentares futuros da criança se a dieta de seus próprios pais for condizente com aquela que preconizam.

Logo, ações sobre o ambiente familiar requerem intervenções sobre um amplo contexto e implicam não só mudanças de hábitos de toda a família, englobando desde refeições à mesa - e não à frente da TV - até prática de atividades físicas em conjunto, sejam elas desportivas, sejam passeios alternativos aos finais de semana. Assim, evita-se que crianças e adolescentes fiquem reclusos e sedentários em casa, assistindo à televisão ou usando o computador.

Quanto ao ambiente escolar, a meta da educação nutricional deve ser a instrução das crianças e adolescentes sobre os princípios gerais de nutrição e alimentação, orientando comportamentos específicos para que estes se tornem aptos a fazerem escolhas conscientes ao longo de suas vidas ${ }^{(8)}$.

Com isso, se no ambiente familiar a promoção de hábitos saudáveis se faz principalmente por certa 'imitação' dos comportamentos dos próprios pais, na escola ocorre a conscientização das escolhas alimentares, por meio da incorporação de conhecimentos básicos de nutrição e do aprendizado da importância de uma boa alimentação para a saúde a longo prazo. Por outro lado, no universo escolar, as crianças e adolescentes deveriam compreender de modo lógico e esclarecido os hábitos alimentares praticados por seus pais e que, tão precocemente, tiveram por referencial; logo, se tornariam aptos a criarem suas próprias opções de cardápio e estilo de vida ${ }^{(9)}$.

Brincar é mais que uma atividade física sem consequências para a criança. Brincando, ela não apenas se diverte, mas também aprende, cria e recria e relaciona-se com o mundo. Desenvolve, ao mesmo tempo, aptidões nos campos cognitivo, linguístico, espacial e visual, corporal e social - como a capacidade de esperar (tolerância), de estabelecer acordos e de criar vínculos afetivos ${ }^{(2)}$.

Por mais educativa que a programação possa ser, a interação com os desenhos e personagens animados é autolimitada. Em geral, as imagens são veiculadas rapidamente e a criança não tem tempo suficiente para compreender parte do que é transmitido, nem para desenvolver um senso crítico ou raciocínio lógico a respeito de tais informações ${ }^{(9)}$.

Assim sendo, como a televisão abrevia parte da criatividade das crianças, os pais devem investir nas brincadeiras, como alternativa extremamente benéfica ao desenvolvimento cognitivo de seus filhos. Afinal, além da função educativa, o ato de brincar pode ser ótimo exercício para o corpo. Percebidas como atividades físicas, as brincadeiras em ambiente aberto atuam como uma importante medida preventiva e mesmo terapêutica contra a obesidade infanto-juvenil e devem, portanto, ser fomentadas tanto em casa como na escola.

Em suma, parece evidente que a introdução da TV no cotidiano das crianças brasileiras tem progressivamente obliterado parte de experiências imprescindíveis para seu desenvolvimento, para o fortalecimento dos laços familiares e mesmo para a criação de vínculos sociais. Logo, é imprescindível que quaisquer ações estimulem uma dieta alimentar equilibrada, associada a brincadeiras e atividades físicas, e fomentem alternativas atraentes e prazerosas que ocupem o tempo de lazer em substituição à televisão e ao computador $^{(9)}$.

\section{As embalagens como meio de comunicação}

Uma embalagem é o recipiente, pacote ou o envoltório destinado a garantir a conservação e facilitar o transporte e manuseio dos alimentos ${ }^{(10)}$. Tradicionalmente, as embalagens para alimentos têm sido planejadas para proteger o produto 
funcionando, assim, como uma barreira inerte entre o alimento e o ambiente ${ }^{(11)}$.

Para o consumidor, é a parte visível do alimento, que traduz a identidade do produto e o fabricante, definindo as reações de vinculação, aceitação ou rechaço do produto. Em muitos casos, é o único meio de comunicação entre o produtor e o consumidor do alimento. Dessa forma, as embalagens apresentam-se como o principal elo de comunicação entre o consumidor, o produto e a marca, de modo que através dela este identifica, escolhe e usa ou não o produto.

No segmento alimentício, utilizar a embalagem e o rótulo para atrair a atenção, aumentar o valor da marca entre os consumidores finais e comunicar os benefícios do produto diretamente na prateleira da loja constitui-se, decerto, em fator de vantagem competitiva ${ }^{(12)}$.

\section{As embalagens: "vendedores mudos"}

Nas últimas décadas, as empresas alimentícias passaram a investir fortemente no design das embalagens de seus produtos, agregando valor a eles ao adequá-los de forma eficiente às necessidades e expectativas do consumidor, dando enfoque especial à praticidade, conveniência, conforto e proteção ao produto. Tais atributos podem ser potencializados pela embalagem graças ao seu alto poder de comunicação, evocando valores emocionais, que também têm reflexos práticos e objetivos como percepção de funcionalidade, identidade e, principalmente, fidelidade à marca ${ }^{(13)}$.

As embalagens, como vendedores mudos, contêm em seu design mensagens visuais diretas, transmitindo significados e imagens que despertam no consumidor a predisposição para aceitação, compra e utilização do produto. Logo, a percepção das informações dependerá de fatores cognitivos e motivacionais do consumidor, dos tipos de códigos utilizados e da forma como são apresentadas na embalagem ${ }^{(12)}$.

\section{O design das embalagens}

Cada vez mais é necessária a ajuda da estética no marketing. Diante de produtos similares, a embalagem é um ponto de diferenciação entre os concorrentes ${ }^{(12)}$. Tendo isso em vista, certos atributos do design agregam valor ao produto e à marca, em termos de praticidade e de conveniência e, principalmente, no sentido de atrair o consumidor, despertar sua atenção e evocar emoções.

A legibilidade em design diz respeito à clareza das palavras na embalagem. O "cartão de visita" de muitos produtos é o seu próprio nome ou sua marca, especialmente quando ambos já estão difundidos no mercado de consumo. Logo, uma boa legibilidade garantirá ao consumidor uma rápida e fácil visualização do alimento desejado na prateleira.

Entretanto, talvez a coloração seja o atributo de maior importância. Utiliza-se a influência da cor e dos seus efeitos psicológicos e sensitivos sobre o consumidor para lançar produtos que agem diretamente sobre esses fatores ${ }^{(12)}$. Em geral, a cor relaciona-se, sobretudo, com a característica do produto. Assim, ela segue determinados padrões que, normalmente, são usados por designers de embalagens. As cores quentes, como o vermelho e amarelo, são aplicadas preferencialmente em embalagens de alimentos, bebidas e redes de fast-food, uma vez que estimulam o sistema nervoso central, abrem o apetite, instigam uma sensação de bem-estar e alegria, destacam-se visualmente e são as mais rapidamente identificadas. Em contrapartida, cores frias como azul, prata e tons pastel são frequentemente usadas em alimentos light, já que provocam a sensação de leveza, equilíbrio, frescor e a diminuição do apetite. Já outras cores, como o verde, são pouco empregadas em guloseimas como biscoitos, porque lembram bolor, dando falsa impressão de que o alimento está estragado ${ }^{(14)}$.

\section{A praticidade das embalagens}

O ritmo acelerado da vida moderna criou um novo consumidor, o qual se caracteriza fundamentalmente pela falta de tempo. As empresas alimentícias incorporaram à estética e funcionalidade de suas embalagens componentes que suprissem essa demanda. As formas tornaram-se mais arrojadas e os tamanhos mais reduzidos, facilitando o transporte e o consumo. As atuais embalagens individuais de sucos naturais, refrigerantes e bebidas lácteas, prontas para o consumo, são facilmente transportadas na bolsa ou na mochila escolar das crianças e adolescentes.

Vistas como alternativas práticas e criativas, pouco se discute sobre as implicações da introdução destas novidades na dieta. Aparentemente inofensivas em seu tamanho reduzido, as porções individuais podem apresentar quantidades significativas de calorias e alto teor de carboidratos, gorduras e sódio. Como o indivíduo come uma menor quantidade do produto por embalagem, ele acaba ingerindo mais porções do alimento por dia; isto é, apesar de o consumo de várias unidades poder levar ao desequilíbrio energético e nutricional da dieta diária ${ }^{(9)}$.

É notória a plasticidade das empresas alimentícias, já que elas costumam renovar a linha de seus produtos no 
sentido de atender às novas demandas. Contudo, a sociedade vem, de forma progressiva, renegando refeições nutritivas e adequadas à saúde em nome da praticidade e da comodidade proporcionadas pela introdução de novas embalagens.

Se esse é um fato irreversível, para evitar danos maiores, as indústrias poderiam produzir embalagens individuais de alimentos nutricionalmente adequados, com mais fibras e menos gorduras, açúcares e sal. Exemplos dessa tendência positiva são as barras de cereais. Fica, no entanto, com a difusão desses hábitos, mesmo com adequação nutricional dos alimentos, não assegurada a gratificação emocional e afetiva do preparo do alimento, da refeição em família e o significado cultural dessa prática ${ }^{(9)}$.

\section{Rótulos}

Rótulo é qualquer informação referente a um produto que esteja transcrita na embalagem. É um elo na comunicação entre produtores e consumidores, principalmente quando se trata da rotulagem de alimentos ${ }^{(15)}$.

Dados recentes demonstraram que $70 \%$ das pessoas consultam rótulos dos alimentos no momento da compra; no entanto, mais da metade não compreende adequadamente o significado das informações. Outra pesquisa, realizada em Brasília, confirma esse resultado: $74,8 \%$ dos pesquisados leem as informações nutricionais, embora apenas $25 \%$ desse grupo tenha o hábito de ler os rótulos de todos os alimentos. Mais da metade desses consumidores que consultava os rótulos lia apenas os referentes a alimentos específicos, com o objetivo de conhecer seu valor calórico. Os resultados obtidos em ambas as pesquisas indicaram que, apesar de a população considerar importante que o rótulo de alimentos contenha as informações nutricionais, a maioria não sabe utilizá-lo ${ }^{(16)}$.

\section{Agência Nacional de Vigilância Sanitária e regulamentações}

Desde 2003, quando se tornou obrigatória a rotulagem nutricional, as informações contidas nos rótulos tornaramse mais complexas. Passou-se a exigir maior habilidade do consumidor para interpretá-las e entendê-las e, consequentemente, ainda mais conhecimento do profissional da saúde para instruir os pacientes e seus familiares.
Quanto à informação nutricional obrigatória, algumas alterações aprovadas pela Resolução da Diretoria Colegiada $(\mathrm{RDC}) \mathrm{n}^{0} 359$ e a $\mathrm{n}^{\mathrm{0}} 360$ foram $^{(17,18)}$ :

- Elementos fundamentais e obrigatórios dos rótulos: lista de ingredientes em ordem decrescente de quantidade utilizada no produto; a origem referente ao fabricante, local de fabricação, etc; prazo de validade; lote para controle da empresa em caso de algum erro na produção, facilitando a localização de todas as outras unidades produzidas conjuntamente; conteúdo líquido expresso em massa ou volume; e informação nutricional obrigatória ${ }^{(17)}$.

- Componentes a serem declarados obrigatoriamente: valor energético, carboidratos, proteínas, gorduras totais, saturadas e trans, fibras alimentares e sódio. A informação nutricional deve ser expressa em porções do alimento ( $g$ ou $\mathrm{mL}$ ) e apresentar medida caseira correspondente, tendo como referência utensílios domésticos (colheres, xícaras ou copos $)^{(17)}$. As vitaminas e os sais minerais, quando presentes em quantidades maiores ou iguais a $5 \%$ da ingestão diária recomendada (IDR) por porção, bem como outros nutrientes considerados relevantes pelo fabricante podem ser declarados opcionalmente. Não obstante, sabe-se que tais nutrientes estão frequentemente presentes nos rótulos e até nas propagandas televisivas de produtos como salgadinhos do tipo snacks e guloseimas. Na maioria das vezes, a informação não é enganosa; entretanto, pode levar a uma interpretação errônea da qualidade nutricional e de supostas vantagens no consumo de alimentos que, como se sabe, devem ser desestimulados na dieta infantojuvenil.

- Valor de referência diária (\%VD) em 2.000kcal: o valor de $2.000 \mathrm{kcal}$, pré-estabelecido como padrão para ser utilizado em rótulos de alimentos embalados (\%VD), não pode ser utilizado como referência para todas as faixas etárias. Por conseguinte, é necessária a observação: \% Valores Diários com base em uma dieta de $2.000 \mathrm{kcal}$ ou $8.400 \mathrm{~kJ}$. Seus valores diários podem ser maiores ou menores dependendo de suas necessidades energéticas ${ }^{(18)}$. Apesar de tal frase contemplar a variabilidade das necessidades nutricionais das crianças segundo sexo, idade e atividade física, o fato é que os pais desconhecem os valores do IDR infanto-juvenis ${ }^{(19)}$. Assim, o \% VD com base em uma dieta de $2.000 \mathrm{kcal}$ acaba se tornando referência, dando uma dimensão incorreta do quão representativo para o consumo diário serão os alimentos. Consomem-se muito mais guloseimas e refrigerantes, por exemplo, sem a idéia de que isto pode facilmente ultrapassar a IDR. 
Portanto, durante a orientação alimentar, é imprescindível que o profissional da saúde enfatize aos pais que nem sempre os percentuais do IDR indicados nos rótulos correspondem à alimentação adequada à idade de seus filhos.

Quanto às informações nutricionais complementares, estas são quaisquer representações que afirmam ou sugerem que um alimento possui uma ou mais propriedades nutricionais particulares relativas ao seu valor energético e/ou seu conteúdo de nutrientes ${ }^{(18,20)}$. Destacam-se:

- Alimentos diet versus alimentos light ${ }^{(20)}$ : os alimentos diet têm formulação especial para atender pessoas que apresentam disfunção e/ou distúrbio físico ou metabólico e necessitam de dietas específicas, como os portadores de diabetes melito, hipertensão e fenilcetonúria. Estes alimentos não devem ter algum determinado ingrediente (açúcar, sódio, glúten, entre outros), o qual será substituído por elementos permitidos em suas dietas, estritamente por razões de saúde. Todavia, isso não significa a redução do valor calórico do alimento em questão. Por outro lado, os alimentos light são aqueles que têm reduzido valor energético, contendo quantidades inferiores a valores pré-fixados pela Agência Nacional de Vigilância Sanitária (Anvisa), conforme o tipo de componente. Assim, há redução de qualquer um de seus macronutrientes, comparados a um produto convencional do mesmo tipo.

- Alimento fonte versus alimento rico ou de alto teor: estes termos normalmente se referem à quantidade de proteínas, fibras alimentares, vitaminas e minerais, nutrientes cujo consumo é desejável e deve ser estimulado. Um alimento fortificado/enriquecido é aquele ao qual foi adicionado um ou mais nutrientes essenciais contidos ou não no alimento, com o objetivo de reforçar o seu valor nutritivo e/ou prevenir ou corrigir deficiência(s) demonstrada(s) em um ou mais nutrientes na alimentação da população ou em grupos específicos ${ }^{(20)}$. Nesse contexto, o que diferencia um alimento fonte de um rico é a quantidade mínima de nutriente (em g ou \% do IDR de referência) em $100 \mathrm{~g}$ ou em $100 \mathrm{~mL}$ do produto, segundo valores pré-fixados pela Anvisa ${ }^{(20)}$. Como propõem os próprios termos, os alimentos de alto teor sempre conterão quantidades superiores de determinado nutriente em relação às fontes. Se o consumidor tiver tal conhecimento, decerto suas escolhas poderão ser feitas com maior critério e propriedade.

- Alimentos probióticos versus prebióticos: probióticos são culturas que estimulam a proliferação de bactérias benéficas da microbiota intestinal, reforçando os me- canismos naturais de defesa do corpo e que, administrados adequadamente, conferem benefícios à saúde ${ }^{(21)}$. $\mathrm{Na}$ indústria alimentícia são encontrados em laticínios como leite fermentado, iogurtes e queijos. Já os prebióticos são carboidratos não-digeríveis que afetam beneficamente o corpo, estimulando seletivamente a proliferação e/ou atividade de bactérias desejáveis no cólon. Utilizados na fabricação de inúmeros alimentos, conferem consistência a produtos lácteos, maciez a produtos de panificação e crocância a biscoitos com baixo teor de gordura $^{(21)}$.

\section{Selos de qualidade}

Em 1991, a Sociedade Brasileira de Cardiologia (SBC) criou o Selo de Aprovação - SBC para certificar a qualidade de alimentos cuja composição nutricional atende às exigências estabelecidas pela comunidade científica para a prevenção dos fatores de risco cardiovasculares, promoção ou manutenção da saúde ou cuja composição não represente risco à saúde ${ }^{(22)}$. Os alimentos candidatos à obtenção do selo submetem-se à criteriosa análise da composição nutricional, em laboratórios credenciados pela Anvisa. Portanto, um produto "aprovado" segue rigorosamente os critérios estabelecidos pelo Comitê do Selo de Aprovação SBC, baseados em padrões nacionais e internacionais de alimentos considerados saudáveis ao coração $^{(22)}$.

A Sociedade Brasileira de Pediatria (SBP) também tem seu selo: o Selo de Certificação da SBP, destinado não só ao segmento alimentício, mas também às indústrias de brinquedos, vestuário, cosméticos e higiene, além de serviços como: escolas de educação infantil, hospitais, profissionais especializados no atendimento de crianças e adolescentes ${ }^{(23)}$.

Tais iniciativas representam uma importante aliança entre profissionais da saúde e indústria alimentícia, sendo uma oportunidade ímpar de órgãos médicos competentes e respeitados avaliarem a qualidade de produtos destinados ao público infanto-juvenil e à sociedade como um todo.

É fato que, principalmente, entre as classes mais abastadas, a preocupação com uma alimentação saudável e seus benefícios a longo prazo criou um novo perfil de consumidor, cada vez mais consciente em relação à importância da qualidade dos produtos que compõem sua mesa. Esses certificados surgem, pois, como alternativas ao desconhecimento da leitura dos rótulos por parcela considerável da sociedade brasileira. Ainda que o consumidor não saiba ler corretamente as informações nutricionais, a presença de um 'selo de garantia' 
emitido por profissionais da área médica é vista como uma 'marca da saúde' de diversos produtos presentes nas prateleiras dos supermercados. Confere-se, portanto, maior credibilidade aos alimentos e fabricantes e, simultaneamente, maior segurança para o consumidor em relação aos benefícios do consumo adequado daquele produto.

\section{Conclusões}

A partir desta abordagem sobre as propagandas de alimentos e sobre o significado das embalagens e dos rótulos e sua importância no momento da compra, é fundamental que os profissionais da saúde saibam lidar com um marketing que, em nome da lucratividade, tem investido pesadamente no design de seus produtos, em prol das novas tendências de consumo de seus consumidores.

A literatura evidencia que os riscos nutricionais e de sedentarismo para o desenvolvimento da obesidade e doenças crônicas associadas são cumulativos. Dessa forma,

\section{Referências bibliográficas}

1. Bleil SI. O padrão alimentar ocidental: considerações sobre a mudança de hábitos no Brasil. Cad Debates 1998;6:1-24.

2. Taddei JA, Brasil AL, Palma D, Moraes DE, Ribeiro LC, Lopez FA et al. Manual crechEficiente: guia prático para educadores e gerentes. São Paulo: Manole; 2006.

3. Serra GMA, Santos EM. Health and media in construction of obesity and perfect body. Ciênc Saúde Coletiva 2003;8:691-701.

4. Linn S, editor. Crianças do consumo: a infância roubada. São Paulo: Instituto Alana; 2006.

5. Almeida S de S, Nascimento PC, Quaioti TC. Amount and quality of food advertisement on Brazilian television. Rev Saude Publica 2002;36:353-5.

6. Halpern G. Comerciais veiculados em programação infanto-juvenil de canais abertos de TV e sua relação com escolha de alimentos em amostra de escolares [dissertação de mestrado]. São Paulo (SP): Unifesp; 2003.

7. Pontes TE, Costa TF, Ferreira AB, Taddei JA, Lopes LA, Brasil AL. Análise da adequação nutricional e sócio-econômica de alimentos veiculados em propagandas durante o horário infantil nas duas emissoras de maior audiência da TV brasileira [iniciação científica]. São Paulo (SP): Unifesp; 2007.

8. Taddei JA. Escolhas conscientes: crianças descobrem os alimentos mais saudáveis com incentivo de jogo educativo. Pesquisa FAPESP [serial on the Internet]. 2004;104 [cited 2006 Oct 16]. Available from: http://www. revistapesquisa. fapesp. $\mathrm{br} /$ ?art $=2600 \& \mathrm{bd}=1 \& \mathrm{pg}=2 \& \mathrm{lg}=$

9. Pontes TE, Feitosa TC, Marum AB, Brasil AL, Taddei JA. Propagandas de alimentos; embalagens; rótulos. In: Palma D, editor. Guia de nutrição: nutrição clínica da criança e do adolescente. Barueri (SP): Manole; 2008. No Prelo.

10. Anvisa - Agência Nacional de Vigilância Sanitária [homepage on the Internet]. Consulta Pública $n^{\circ} 71$, de 10 de novembro de 2006 [cited 2007 Dec 13]. Available from: http://www4.anvisa.gov.br/base/visadoc/CP/CP\%5B165561-0\%5D.PDF

11. Azeredo HMC, Faria JA, Azeredo AMC. Embalagens ativas para alimentos. Ciênc Tecnol Aliment Campinas 2000;20:337-41.

12. Silveira Neto WD. Avaliação visual de rótulos de embalagens [dissertação de mestrado]. Florianópolis (SC): Universidade Federal de Santa Catarina; 2001. as gerações de crianças nascidas em ambiente que estimula o sedentarismo e o consumo excessivo e rotineiro de alimentos pouco saudáveis acumulam riscos desde o nascimento. Estarão, portanto, mais propensos a desenvolverem doenças crônicas limitantes mais precocemente. Por esses motivos, torna-se urgente a adoção de práticas preventivas com esclarecimento aos familiares, visando minimizar tais efeitos.

Logo, o conhecimento sobre a composição nutricional de tais alimentos, as regras de rotulagem e embalagem, assim como as técnicas de propaganda e marketing são, de fato, indispensáveis para que o profissional de saúde infantil possa alertar e orientar adequadamente seus pacientes e familiares na prática clínica.

\section{Agradecimentos}

Ao Conselho Nacional de Desenvolvimento Científico e Tecnológico (CNPq) e ao Instituto Alana - Projeto Criança e Consumo.
13. Associação Brasileira de Embalagens [homepage on the Internet]. Comitê de design: o valor do design [cited 2006 Dec 15]. Available from: http:// comitedesign.abre.org.br/

14. FURG - Fundação Universidade Federal do Rio Grande [homepage on the Internet]. Portal de embalagens [cited 2006 Dec 15]. Available from: http:// www.furg.br/portaldeembalagens/

15. Anvisa - Agência Nacional de Vigilância Sanitária [homepage on the Internet] Gerência Geral de Alimentos. Universidade de Brasília, Departamento de Nutrição. Manual de orientação aos consumidores: educação para o consumo saudável. Brasília: Ministério da Saúde; 2005 [cited 2007 Nov 22]. Available from: http://www.anvisa.gov.br/ALIMENTOS/rotulos/manual_consumidor.pdf

16. Monteiro RA, Coutinho JG, Recine E. Consulta aos rótulos de alimentos e bebidas por freqüentadores de supermercados em Brasília, Brasil. Rev Panam Salud Publica 2005;18:172-7.

17. Anvisa - Agência Nacional de Vigilância Sanitária [homepage on the Internet]. Resolução RDC n 359, de 23 de dezembro de 2003 [cited 2007 Jan 30]. Available from: http://e-legis.anvisa.gov.br/leisref/public/showAct.php?id=9058

18. Anvisa - Agência Nacional de Vigilância Sanitária [homepage on the Internet]. Resolução RDC n 360, de 23 de dezembro de 2003. [cited 2007 Jan 30]. Available from: http://www.anvisa.gov.br/legis/resol/2003/rdc/360_03rdc.htm

19. IOM - Institute of Medicine [homepage on the Internet]. Dietary intake 2001/ 2002 [cited 2006 Sep 27]. Available from: http://www.iom.edu/Object.File/ Master/21/372/0.pdf

20. Anvisa - Agência Nacional de Vigilância Sanitária [homepage on the Internet] Portaria $n^{\circ} 27$, de 13 de janeiro de 1998 [cited 2007 Jan 30]. Available from: http://e-legis.anvisa.gov.br/leisref/public/showAct.php?id=97\&word=

21. Saad SM. Probiotics and prebiotics: the state of the art. Rev Bras Cienc Farm 2006;42:1-16.

22. SBC - Sociedade Brasileira de Cardiologia [homepage on the Internet]. Selo de Aprovação SBC [cited 2007 Jun 9]. Available from: http://prevencao.cardiol. $\mathrm{br} /$ selodeaprovacao/

23. SBP - Sociedade Brasileira de Pediatria [homepage on the Internet]. Selo da SBP [cited 2007 Jun 9]. Available from: http://www.sbp.com.br/show_item. cfm?id_categoria $=28 \&$ tipo $=$ I 